temperature the iron oxide sinters and the emission falls and becomes unstable. This temperature limitation defines the maximum emission that can be taken from an iron oxide source with a given metal on it. The higher the work function for the positive ions the lower the maximum current. In the mobility experiments with gallium we must have exceeded this limiting temperature in the attempt to obtain the necessary emission.

Details of these experiments, together with others on the properties of surface ionic layers of other metals on various oxides, will be discussed in another place. We may point out here the significance of these results in connexion with the catalytic action of the Kunsman source in the production of ammonia which we have found to take place at low pressures ${ }^{3}$ and which is used as an industrial process. The catalytic activity may be assumed to be directly associated with the presence of alkali ions on an enormous area, and any activity of the oxide in the absence of added alkali to the inevitable presence of traces of the alkalis as impurity, for a small mass of alkali will cover a large area as a monatomic film.

C. F. Powell. Luang Brata.

The Wills Physical Laboratory, University of Bristol. Dec. 20.

Tyndall and Powell, Proc. Roy. Soc., A, 136, $145 ; 1932$.
2 Lennard-Jones, Trans. Faraday Soc., 28, 333; 1932. 3 Powell and Brata, Proc. Roy. Soc., A, 138, 117 ; 1932.

\section{Fusion of Carbon}

In the course of an investigation on the behaviour of the carbon arc in various gases at high pressure, carried out in apparatus provided by Imperial Chemical Industries Ltd., we have obtained large amounts of carbon in a fine state of division. In certain circumstances this carbon is deposited as graphite, all in the form of small spherules of about $0.02 \mathrm{~mm}$. diameter or smaller, having a density $\mathbf{2} \cdot 25$. There seems to be little doubt that these spherules of graphite have been formed owing to the effect of surface tension, the carbon having persisted for a finite time in the molten state. The best yield was obtained at 90 atmospheres in hydrogen with an are dissipating 20 kilowatts. The temperature of the positive crater at this pressure has not yet been determined. The surface brightness at 10.5 atmospheres was $2 \cdot 18$ times that of the arc at atmospheric pressure for the same wave-length, corresponding to an increase of brightness temperature of $460^{\circ}$. The increase in the thermodynamic temperature must be of the same order, so that the temperature was probably much higher at 90 atmospheres. Further investigations are necessary before the precise conditions under which the spherules are formed are established. The percentage of ash left after burning the carbon of the electrodes was $0 \cdot 8$, that from the carbon collected from the arc chamber less than 3 per cent. It is scarcely likely that this amount of fusible ash could cause the whole of the carbon to adopt spherical form.

Braun $^{1}$ has mentioned the formation of microscopic spheres of carbon from thin carbon filaments through which the discharge from a number of Leyden jars was passed, while Lummer's investigations on the peculiar molten appearance of the positive crater in arcs at low pressure described in his book on the "Verflussigung der Kohle" are well known, but no evidence of the melting of carbon is quite so convincing as the appearance of these graphite spherules, obtained in the above manner.

A. Egerton.

Clarendon Laboratory,

M. MILFord.

Oxford.

Dec. 15.

${ }^{1}$ Ann. Phys., 17, 359; 1905.

\section{Liesegang Rings}

IN reply to Dr. Bradford's letter ${ }^{1}$ I would say that the more comprehensive a theory is, the more general and apparently indefinite must be the terms in which it is expressed. If for my indefinite term 'mobilisa. tion' is substituted the more definite term 'adsorption', my theory of periodic structures becomes almost identical with Dr. Bradford's. But I believe that adsorption is only one of the means by which the substance may migrate towards the structure already formed, and I prefer, therefore, to use the less definite term 'mobilisation' to include all these means.

It is not correct to suppose that in accordance with my theory all systems should give periodic structures, for the critical conditions postulated may be absent or only slightly developed. Nevertheless, it is probable that a system not usually given to periodic structure formation can always be made to furnish periodic structures by alteration of these conditions. This, I believe, is a point also upheld by Dr. Bradford.

International Tin Research ERnest S. Hedges. and Development Council, Manfield House, Strand, London, W.C.2. Jan. 4.

1 Nature, 130, 1002, Dec. 31, 1932.

\section{Nitrogen Fixation in the Genus Lolium}

THE results of some recent experiments suggest that it is highly probable that part of the nitrogen requirement of plants of the genus Lolium is met from atmospheric sources.

Experiments were designed to test the effect of withholding atmospheric nitrogen from the plants. The plants were grown in sterile silver sand, which had previously been freed of all traces of nitrogen. The containers for the sand were large specimen tubes. There were two separate series of these sand cultures, one supplied with combined nitrate nitrogen, the other without it. Two sterilised seeds were buried in each tube, four tubes of each type were placed under a bell jar which was made gas-tight, and a mixture of 80 per cent hydrogen, 20 per cent oxygen and 0.03 per cent carbon dioxide was drawn through. A control series of the same types and prepared under the same experimental conditions was run simultaneously under a bell jar through which atmospheric air was drawn. During these early experiments the gas mixture of both chambers was drawn through the sand. The gas in the chambers was changed once every twenty-four hours. The hydrogen and oxygen were prepared electrolytically, and the carbon dioxide by the action of hydro. chloric acid on marble chips.

The experiment was kept going for seven weeks. 